Real Analysis Exchange

Vol. 23(2), 1997-1998, pp. 459-469

Dariusz Doliwa and Ryszard Jerzy Pawlak, Faculty of Mathematics, Łódź

University, Banacha 22, 90-238 Łódź, Poland,

e-mail:rpawlak@imul.uni.lodz.pl

\title{
ON THE ALMOST CONTINUITY OF THE DIAGONAL OF FUNCTIONS
}

\begin{abstract}
In this paper we consider the following questions: Under what additional assumptions is the diagonal $f \triangle g$ of almost continuous functions vanishing on the set of discontinuity points an almost continuous function, too. Moreover, one can show that the facts considered (diagonals and ac-homotopies) can be applied to the characterization of continuity or the investigations of the algebraic operations on almost continuous functions.
\end{abstract}

Almost continuous functions, introduced by Stalling, ([8]) (in order to generalize the Brouwer fixed point theorem) have been intensively studied by many authors. There are various areas of interest in this study. Among them are the problems connected with the operations on almost continuous functions. It has been well known for several years that if $f$ is an almost continuous function and $g$ is a continuous function, then the diagonal function $f \triangle g$ is an almost continuous function ([6], [4]). In [6] the author proved the following theorem: Suppose that $Y_{0}$ and $Z_{0}$ are convex subsets of normed spaces $Y$ and $Z$, respectively, $f_{1}: \mathbb{I} \rightarrow Y_{0}, f_{2}: \mathbb{I} \rightarrow Z_{0}$ are almost continuous functions and $D$ is the set of points at which $f_{1}$ is discontinuous. If the restriction of $f_{1 \mid \bar{D}}$ is continuous and each point of $\bar{D}$ is a point of continuity of $f_{2}$, then the diagonal $f_{1} \triangle f_{2}$ is an almost continuous function.

In our paper we investigate the following question: Under what additional assumptions is the diagonal of almost continuous functions also an almost continuous function, even if the sets of discontinuity points are not disjoint.

We use the standard notions and notation. The open ball with center at $x$ and radius $r>0$ will be denoted by $B(x, r)$. The symbols $\bar{A}, \operatorname{Fr} A$ and $\operatorname{Int}(A)$ stand for the closure, the boundary and the interior of $A$, respectively.

Key Words: diagonal function; almost continuity; porosity.

Mathematical Reviews subject classification: 26A15; 54C08

Received by the editors March 24, 1997 
By $\mathbb{R}(\mathbb{I})$ we denote the set of all real numbers (the segment $[0,1]$ ) with the natural topology $\tau_{o}$. By $C_{f}\left(D_{f}\right)$ we shall denote the set of all continuity (discontinuity) points of $f$. The symbol $\Gamma(f)$ stands for the graph of $f$. The restriction of $f$ to the set $A$ is denoted by $f_{\mid A}$. For a function $f: X \rightarrow \mathbb{R}^{n}$, we put $\mathcal{Z}(f)=\{x \in X: f(x)=(0,0, \ldots, 0)\}$.

A function $f: X \rightarrow Y$ where $X, Y$ are topological spaces is almost continuous if, for each open set $U \subset X \times Y$ containing $\Gamma(f), U$ contains the graph of some continuous functions $g: X \rightarrow Y$. The set of all almost continuous functions mapping $X$ into $Y$ (as well as the space ${ }^{1}$ with the metric of uniform convergence $\varrho$ ) will be denoted by $\mathcal{A}(X, Y)$.

In this paper we consider functions belonging to the set

$$
\mathcal{A}_{o}\left(X, \mathbb{R}^{n}\right)=\left\{f \in \mathcal{A}\left(X, \mathbb{R}^{n}\right): D_{f} \subset \mathcal{Z}(f)\right\}
$$

and

$$
\mathcal{D}_{o}\left(X, \mathbb{R}^{n}\right)=\left\{f \in \mathcal{D}\left(X, \mathbb{R}^{n}\right): D_{f} \subset \mathcal{Z}(f)\right\}
$$

where $n \in 1,2)$ and $\mathcal{D}\left(X, \mathbb{R}^{n}\right)$ is a family of Darboux functions ${ }^{2}$ mapping $X$ into $\mathbb{R}$.

For functions $f: X \rightarrow Y$ and $g: X \rightarrow Z$, we define their diagonal function $f \triangle g: X \rightarrow Y \times Z$ by $(f \triangle g)(x)=(f(x), g(x))$.

Let $f \in \mathcal{A}(X, Y)\left(f \in \mathcal{A}_{o}(X, Y)\right)$. Then we define $\Delta_{\mathcal{A}}(f)\left(\Delta_{\mathcal{A}_{o}}(f)\right)$ by

$$
\begin{aligned}
\Delta_{\mathcal{A}}(f) & =\{g \in \mathcal{A}(X, Y): f \triangle g \in \mathcal{A}(X, Y \times Y)\} \\
\left(\Delta_{\mathcal{A}_{o}}(f)\right. & \left.=\left\{g \in \mathcal{A}_{o}(X, Y): f \triangle g \in \mathcal{A}_{o}(X, Y \times Y)\right\}\right) .
\end{aligned}
$$

Let $(X, \tau),\left(X, \tau^{\prime}\right)$ be topological spaces. We say that the topology $\tau^{\prime}$ is s-finer than $\tau$ if $\tau^{\prime}$ is finer than $\tau$ and $\{U \in \tau: U$ is $\tau$-closed $\}=\{U \in \tau$ : $U$ is $\tau^{\prime}$-closed $\}$. In a similar way as in [7] we can define ac-homotopies: The almost continuous functions $f, g:(X, \tau) \rightarrow\left(X, \tau^{*}\right)$ are called ac-homotopic if there exists a topology $\tau^{\prime}$ s-finer than the topology $\tau$, such that $f, g:\left(X, \tau^{\prime}\right) \rightarrow$ $\left(Y, \tau^{*}\right)$ are continuous and there exists a homotopy $\xi:\left(X, \tau^{\prime}\right) \times \mathbb{I} \rightarrow\left(Y, \tau^{*}\right)$ between $f$ and $g$ such that $\xi:(X, \tau) \times \mathbb{I} \rightarrow\left(Y, \tau^{*}\right)$ is an almost continuous function. (The fact that $f$ and $g$ are ac-homotopic and $\xi$ is an ac-homotopy between $f$ and $g$ is written down as $f \frac{a c}{\xi} g$.)

The notions and symbols we use, connected with porosity, come from papers [9] and [10]. Let $X$ be a metric space. Let $M \subset X, x \in X$ and

\footnotetext{
${ }^{1}$ Of course, in this case we consider only bounded functions.

${ }^{2} \mathrm{~A}$ function $f: X \rightarrow Y$ is a Darboux function if the image of any connected set is a connected set, too.
} 
$R>0$. Then we denote by $\gamma(x, R, M)$ the supremum of the set of all $r>0$ for which there exists $z \in X$ such that $B(z, r) \subset B(x, R) \backslash M$. If $p(M, x)=2 \cdot \lim \sup _{R \rightarrow 0+} \frac{\gamma(x, R, M)}{R}>0$, then we say that $M$ is porous at $x$.

Before giving the main results, we make the following observation.

Remark. If $f: \mathbb{R} \rightarrow \mathbb{R}$ is a function such that $D_{f} \subset \mathcal{Z}(f)$, then $D_{f}$ is nowhere dense set.

The example given below ${ }^{3}$ shows that there exist two functions $f, g \in$ $\mathcal{A}_{o}(\mathbb{R}, \mathbb{R})$ such that $D_{f}=\{0\}=D_{g}$ and $f(0)=0=g(0)$ but $f \triangle g$ is not an almost continuous function.

Example. Let $f(x)=0$ for $x \in(-\infty, 0] \cup[1,+\infty) \cup\left\{\frac{1}{2 n+1}: n=0,1,2, \ldots\right\}$; $f\left(\frac{1}{2 n}\right)=1(n=1,2, \ldots)$ and let $f$ be linear in each interval $\left[\frac{1}{n+1}, \frac{1}{n}\right](n=$ $1,2, \ldots)$. Then $D_{f}=\{0\}$ and, consequently ( $f$ is a Darboux, Baire one function), $f \in \mathcal{A}_{o}(\mathbb{R}, \mathbb{R})([2])$. In each interval $\left[\frac{1}{2 n+2}, \frac{1}{2 n+1}\right]\left(\left[\frac{1}{2 n+3}, \frac{1}{2 n+2}\right]\right.$ ) (for $n=0,1,2, \ldots)$ we can choose a point $p_{n}\left(q_{n}\right)$ such that $f\left(p_{n}\right)=\frac{3}{4}\left(f\left(q_{n}\right)=\frac{3}{4}\right)$ $(n=0,1,2, \ldots)$.

Now, we define a function $g: \mathbb{R} \rightarrow \mathbb{R}$ in the following way: $g(x)=0$ for $x \in(-\infty, 0] \cup[1,+\infty) \cup\left\{\frac{1}{2 n}: n=1,2, \ldots\right\} ; g\left(\frac{1}{2 n+1}\right)=1(n=1,2, \ldots)$; $g\left(p_{n}\right)=g\left(q_{n}\right)=\frac{3}{4}$ for $n=0,1,2, \ldots$; let $g$ be a linear function in each interval $\left[p_{n}, \frac{1}{2 n+1}\right],\left[\frac{1}{2 n+2}, p_{n}\right],\left[q_{n}, \frac{1}{2 n+2}\right],\left[\frac{1}{2 n+3}, q_{n}\right]$ for $n=0,1,2, \ldots$

It is easy to see that $f, g \in \mathcal{A}_{o} ; f \triangle g(0)=(0,0)$ and $f \triangle g(x) \notin\left(-\frac{1}{2}, \frac{1}{2}\right) \times$ $\left(-\frac{1}{2}, \frac{1}{2}\right)$ for $x \in\left(0, \frac{1}{2}\right]$ and so, $f \triangle g\left(\left[0, \frac{1}{2}\right]\right)$ is not a connected set, which means that $f \triangle g \notin \mathcal{D}\left(\mathbb{R}, \mathbb{R}^{2}\right.$ ) and, consequently (cf. [6], Theorem 1.1.11), f $\triangle g \notin$ $\mathcal{A}\left(\mathbb{R}, \mathbb{R}^{2}\right)$.

The problem raised at the beginning can be formulated in the following way: Given a fixed function $f \in \mathcal{A}_{o}(\mathbb{R}, \mathbb{R})$, under what assumptions on $g \in$ $\mathcal{A}_{o}(\mathbb{R}, \mathbb{R})$ do we have $g \in \Delta_{\mathcal{A}_{o}}(f)$ ? The answer is contained in the next theorem which we precede by the following lemma.

Lemma 1. $\mathcal{A}_{o}\left(\mathbb{R}, \mathbb{R}^{n}\right)=\mathcal{D}_{o}\left(\mathbb{R}, \mathbb{R}^{n}\right)$.

Proof. The inclusion $\quad \mathcal{A}_{o}\left(\mathbb{R}, \mathbb{R}^{n}\right) \quad \subset \quad \mathcal{D}_{o}\left(\mathbb{R}, \mathbb{R}^{n}\right)$ follows from Theorem 1.1.11 of paper [6].

Now, we shall prove the opposite inclusion. So, let $f \in \mathcal{D}_{o}\left(\mathbb{R}, \mathbb{R}^{n}\right)$. Note that $\overline{D_{f}}$ is closed and nowhere dense set and $f$ is continuous on every component of $\mathbb{R} \backslash \overline{D_{f}}$. Since $f$ is Darboux function, $f$ is almost continuous on the closure of every component of $\mathbb{R} \backslash \overline{D_{f}}$ (see [6], Theorem 1.5.2) and according to ([6], Lemma 1.3.2), $f$ is almost continuous.

\footnotetext{
${ }^{3}$ This example is very simple. However, the functions constructed within the framework of it will be useful in the explanation of certain questions in the remainder of the paper.
} 
Theorem 1. Let $f \in \mathcal{A}_{o}(\mathbb{R}, \mathbb{R})$. Then $g \in \Delta_{\mathcal{A}_{o}}(f)$ if and only if $f \frac{a c}{\xi} g$ and $\left(\overline{D_{f}} \cup \overline{D_{g}}\right) \times \mathbb{I} \subset \mathcal{Z}(\xi)$, for an ac-homotopy $\xi$.

Proof. Necessity. Put $D=D_{f} \cup D_{g}$. It is not hard to verify that

$$
D=D_{f \triangle g} \text { and } \bar{D} \subset \mathcal{Z}(f \triangle g) .
$$

Set $D^{\prime}=\mathbb{R} \backslash \bar{D}$ and let $\left\{\left(p_{n}, q_{n}\right)\right\}_{n}$ be the sequence of all components of the set $D^{\prime}$. (It is possible that $p_{n}=-\infty$ or $q_{n}=+\infty$ for some n.) Fix $x \in D^{\prime}$. Then there exists a positive integer $n_{x}$ such that $x \in\left(p_{n_{x}}, q_{n_{x}}\right)\left(p_{n_{x}}<q_{n_{x}}\right)$. Put

$$
\mathcal{B}(x)=\left\{(x-\delta, x+\delta): \delta \in\left(0, \min \left(x-p_{n_{x}}, q_{n_{x}}-x\right)\right)\right\} .
$$

Now, let $x \in \bar{D}$ and let $k$ be a fixed positive integer. Consider the interval $\left(x, x+\frac{1}{k}\right)$, and let $U_{x}^{k}=\left(x, x+\frac{1}{k}\right) \cap D^{\prime}$. According to the Remark above, $U_{x}^{k}$ is an open and dense set in $\left[x, x+\frac{1}{k}\right]$.

Now, we shall show that

$$
\text { there exists } z_{x}^{k} \in U_{x}^{k} \text { such that } f\left(z_{x}^{k}\right), g\left(z_{x}^{k}\right) \in\left(-\frac{1}{k}, \frac{1}{k}\right) \text {. }
$$

Indeed, suppose to the contrary that, for each $y \in U_{x}^{k}, f(y) \notin\left(-\frac{1}{k}, \frac{1}{k}\right)$ or $g(y) \notin\left(-\frac{1}{k}, \frac{1}{k}\right)$. Therefore $f \triangle g\left(U_{x}^{k}\right) \cap\left(\left(-\frac{1}{k}, \frac{1}{k}\right) \times\left(-\frac{1}{k}, \frac{1}{k}\right)\right)=\emptyset$ and, consequently, $f \triangle g\left(\left[x, x+\frac{1}{k}\right]\right) \subset\{(0,0)\} \cup\left(\mathbb{R}^{2} \backslash\left(\left(-\frac{1}{k}, \frac{1}{k}\right) \times\left(-\frac{1}{k}, \frac{1}{k}\right)\right)\right)$ and, moreover (according to (1)),

$(0,0) \in f \triangle g\left(\left[x, x+\frac{1}{k}\right]\right)$ and $\left(\mathbb{R}^{2} \backslash\left(\left(-\frac{1}{k}, \frac{1}{k}\right) \times\left(-\frac{1}{k}, \frac{1}{k}\right)\right)\right) \cap f \triangle g\left(\left[x, x+\frac{1}{k}\right]\right) \neq \emptyset$.

This means that $f \triangle g$ is not a Darboux function and, consequently (cf. Theorem 1.1.11 of [6]), $f \triangle g \notin \mathcal{A}\left(\mathbb{R}, \mathbb{R}^{2}\right)$, which contradicts our assumption. The proof of (2) is finished.

Of course, $z_{x}^{k} \in D^{\prime}$. Let $t_{z_{x}^{k}}$ be a positive integer such that $\left(z_{x}^{k}-\frac{1}{t_{z_{x}^{k}}}, z_{x}^{k}+\right.$ $\left.\frac{1}{t_{z_{x}^{k}}}\right)$ is a subset of the component of $U_{x}^{k}$, containing $z_{x}^{k}$ and $f\left(\left(z_{x}^{k}-\frac{1}{t_{z_{x}^{k}}}, z_{x}^{k}+\right.\right.$ $\left.\left.\frac{1}{t_{z_{x}^{k}}}\right)\right) \subset\left(-\frac{1}{k}, \frac{1}{k}\right) \supset g\left(\left(z_{x}^{k}-\frac{1}{t_{z_{x}^{k}}}, z_{x}^{k}+\frac{1}{t_{z_{x}^{k}}}\right)\right)$. By the arbitrariness of $k$ and $x \in \bar{D}$, we can construct the set $U_{x}^{k}$ for any positive integer $k$ and $x \in \bar{D}$. Additionally, we may assume that

$$
\text { if } k_{1}<k_{2} \text {, then } z_{x}^{k_{1}}>z_{x}^{k_{2}} \text { for any } x \in \bar{D} .
$$

Put

$$
U_{s}^{+}(x)=\{x\} \cup \bigcup_{k=s}^{\infty}\left(z_{x}^{k}-\frac{1}{t_{z_{x}^{k}}+s}, z_{x}^{k}+\frac{1}{t_{z_{x}^{k}}+s}\right) \text { for } s=1,2, \ldots \text { and } x \in \bar{D} .
$$


In a similar way we can define the sets $U_{s}^{-}(x)$. Let

$$
\mathcal{B}(x)=\left\{U_{s}(x)=U_{s}^{-}(x) \cup U_{s}^{+}(x): s=1,2, \ldots\right\} \text { for } x \in \bar{D} .
$$

Let $\tau$ be a topology generated by the local system $\{\mathcal{B}(x)\}_{x \in \mathbb{R}}$. It is easy to see that $\tau$ is finer than $\tau_{o}$. Now, we shall show that

$$
\tau \text { is } s \text {-finer than } \tau_{o} \text {. }
$$

To prove this fact, it is sufficient to show that $\left\{V \in \tau_{o}: V\right.$ is $\tau$-closed $\}=$ $\{\emptyset, \mathbb{R}\}$. Suppose to the contrary that there exists a $\tau$-closed set $V_{o} \in \tau_{o}$ such that $\emptyset \neq V_{o} \neq \mathbb{R}$. Let $(u, v)(u<v)$ be a component of $V_{o}$ (in the topology $\left.\tau_{o}\right)$. Suppose, for instance, that $u>-\infty$. From the construction of $\mathcal{B}(u)$ (in the case when $u \in D^{\prime}$, as well as in the case when $u \in \bar{D}$ ) we may deduce that $u \in \operatorname{cl}_{\tau}((u, v))$ where $\operatorname{cl}_{\tau}$ denotes the closure in the space $(\mathbb{R}, \tau)$. Then $u \in \operatorname{cl}_{\tau}\left(V_{o}\right)$ and, consequently ( $V_{o}$ is a $\tau$-closed set $), u \in V_{o}$, which is impossible because $(u, v)$ is a component of $V_{o}$ (in the topology $\tau_{o}$ ).

Let us observe that

$$
f, g:(\mathbb{R}, \tau) \rightarrow \mathbb{R} \text { are continuous functions. }
$$

Now, let $\xi:(\mathbb{R}, \tau) \times \mathbb{I} \rightarrow \mathbb{R}$ be a natural homotopy between $f$ and $g$, which means that

$$
\xi(x, r)=(1-r) f(x)+r g(x) \text { for } x \in \mathbb{R}, r \in \mathbb{I} .
$$

From (1) we conclude that

$$
\left(\overline{D_{f}} \cup \overline{D_{g}}\right) \times \mathbb{I} \subset \mathcal{Z}(\xi) .
$$

The proof of necessity will be completed by showing that

$$
\xi: \mathbb{R} \times \mathbb{I} \rightarrow \mathbb{R} \text { is an almost continuous; function }
$$

(in the natural topology of $\mathbb{R} \times \mathbb{I}$ ). Let $\left\{a_{m}\right\}_{-\infty}^{+\infty} \subset \operatorname{Int}\left(D^{\prime}\right)$ be a sequence of real numbers such that

$$
-\infty \leftarrow \cdots<a_{-2}<a_{-1}<a_{o}<a_{1}<a_{2}<\cdots \rightarrow+\infty .
$$

Let $V$ be an arbitrary open set containing $\Gamma(\xi)$. Fix a positive integer $m$ and put $I_{m}=\left[a_{m}, a_{m+1}\right]$. First, we assume that $I_{m} \cap \bar{D} \neq \emptyset$. It is not hard to verify that

$$
\begin{array}{r}
\forall_{x \in \bar{D} \cap I_{m}} \exists_{\delta_{x}>0}\left(\left[x-\delta_{x}, x+\delta_{x}\right] \times \mathbb{I}\right) \times\left[-\delta_{x}, \delta_{x}\right] \subset V \\
\text { and }\left[x-\delta_{x}, x+\delta_{x}\right] \subset \operatorname{Int} I_{m} .
\end{array}
$$


In the rest of the paper, $\delta_{x}$ always denotes fixed positive numbers for $x \in$ $\bar{D} \cap I_{m}$, such that relations (5) take place.

Now, for $x \in \bar{D} \cap I_{m}$, let $k_{x}$ denote positive integers such that $\overline{U_{k_{x}}} \subset$ $\left(x-\delta_{x}, x+\delta_{x}\right)$ and $f\left(U_{k_{x}}\right) \subset\left(-\frac{\delta_{x}}{2}, \frac{\delta_{x}}{2}\right) \supset g\left(U_{k_{x}}\right) ; U_{k_{x}}=U_{k_{x}}(x) \in \mathcal{B}(x)$. This means that

$$
\forall_{x \in \bar{D} \cap I_{m}} \xi\left(U_{k_{x}} \times \mathbb{I}\right) \subset\left(-\delta_{x},+\delta_{x}\right) .
$$

We let $x^{\prime}=\inf U_{k_{x}} \in \operatorname{Int}\left(I_{m}\right), x^{\prime \prime}=\sup U_{k_{x}} \in \operatorname{Int}\left(I_{m}\right)$ for $x \in \bar{D} \cap I_{m}$. Now, we consider the family $\left\{D^{\prime} \cap I_{m}\right\} \cup\left\{\left(x^{\prime}, x^{\prime \prime}\right) ; x \in \bar{D} \cap I_{m}\right\}$. Let $x_{1}, x_{2}, \ldots, x_{q}$ be a finite sequence of numbers belonging to $\bar{D} \cap I_{m}$ such that

$$
I_{m}=\left(D^{\prime} \cap I_{m}\right) \cup \bigcup_{n=1}^{q}\left(x_{n}^{\prime}, x_{n}^{\prime \prime}\right)
$$

Without loss of generality we may assume that

$$
x_{1}^{\prime} \leq x_{2}^{\prime} \leq \ldots .
$$

Put $\delta_{o}=\min \left(\delta_{x_{1}}, \delta_{x_{2}}, \ldots, \delta_{x_{q}}\right)$. First, we consider $x_{1}$ and components $\left(x_{1}^{\prime}, s_{1}\right)$ and $\left(z_{1}, x_{1}^{\prime \prime}\right)$ of the set $U_{k_{x_{1}}}$. Let $x_{*}^{1} \in\left(x_{1}^{\prime}, s_{1}\right)$. From (6) we infer that $\xi\left(\left\{x_{*}^{1}\right\} \times\right.$ II) $\subset\left(-\delta_{x_{1}}, \delta_{x_{1}}\right)$. Let $x_{1}^{*} \in\left(z_{1}, x_{1}^{\prime \prime}\right)$. Then $\xi\left(\left\{x_{1}^{*}\right\} \times \mathbb{I}\right) \subset\left(-\delta_{x_{1}}, \delta_{x_{1}}\right)$. If $x_{1}^{\prime \prime}$ belongs to the component of $D^{\prime}$ which contains $a_{m+1}$, then we end our considerations. If not, we shall consider $x_{2}$. If $x_{2}^{\prime \prime} \leq x_{1}^{\prime \prime}$, then we are passing to the next step dealing with the considerations of $x_{3}$. So, we suppose that $x_{2}^{\prime \prime}>x_{1}^{\prime \prime}$. Then, according to (7), (8), $x_{2}^{\prime}<x_{1}^{\prime \prime}$ or $x_{2}^{\prime \prime}$ belongs to the component of $D^{\prime}$ which contains $x_{1}^{\prime \prime}$.

Let $\left(d_{2}^{\prime}, d_{2}^{\prime \prime}\right)$ be a component of $D^{\prime}$ such that $x_{1}^{\prime \prime} \in\left[d_{2}^{\prime}, d_{2}^{\prime \prime}\right]$. Since $x_{1}^{\prime \prime}$ does not belong to the component of $D^{\prime}$ which contains $a_{m+1}$, we may infer that $d_{2}^{\prime \prime}<a_{m+1}$. Denote by $x_{*}^{2}$ a point from the interval $\left(\max \left(x_{1}^{*}, x_{2}^{\prime}\right), d_{2}^{\prime \prime}\right)$ such that $f\left(x_{*}^{2}\right) \in\left(-\frac{\delta_{o}}{2}, \frac{\delta_{o}}{2}\right)$ and $g\left(x_{*}^{2}\right) \in\left(-\frac{\delta_{o}}{2}, \frac{\delta_{o}}{2}\right)$. Then $\xi\left(\left\{x_{*}^{2}\right\} \times \mathbb{I}\right) \subset\left(-\delta_{o}, \delta_{o}\right) \subset$ $\left(-\delta_{x_{2}}, \delta_{x_{2}}\right)$.

Now, we consider the component $\left(b_{2}, x_{2}^{\prime \prime}\right)$ of $U_{k_{x_{2}}}$. Let $x_{2}^{*} \in\left(z_{2}, x_{2}^{\prime \prime}\right)$. Then $\xi\left(\left\{x_{2}^{*}\right\} \times \mathbb{I}\right) \subset\left(-\delta_{x_{2}}, \delta_{x_{2}}\right)$. Now, if $x_{2}^{*}$ belongs to the component of $D^{\prime}$ which contains $a_{m+1}$, then we end our considerations. In the opposite case, we shall consider $x_{3}$. There is no loss of generality in assuming that we obtain the sequence

$$
x_{o}^{*}=a_{m}<x_{*}^{1}<x_{1}^{*}<x_{*}^{2}<x_{2}^{*}<\cdots<x_{*}^{q}<x_{q}^{*}<a_{m+1}=x_{*}^{q+1} .
$$

It is easy to see that

$$
\forall_{i=1,2, . ., q}\left[x_{*}^{i}, x_{i}^{*}\right] \subset\left(x_{i}-\delta_{x_{i}}, x_{i}+\delta_{x_{i}}\right) \text { and } \xi\left(\left\{x_{*}^{i}, x_{i}^{*}\right\} \times \mathbb{I}\right) \subset\left(-\delta_{x_{i}}, \delta_{x_{i}}\right) .
$$


Let $\xi_{i}=\xi_{\mid\left\{x_{*}^{i}, x_{i}^{*}\right\} \times \mathbb{I}}:\left\{x_{*}^{i}, x_{i}^{*}\right\} \times \mathbb{I} \rightarrow\left[-\delta_{x_{i}}, \delta_{x_{i}}\right]$ for $i=1,2, \ldots, q$. Then $\xi_{i}$ is a continuous function $(i=1,2, \ldots, q)$ and so there exists a continuous extension $\xi_{i}^{*}:\left[x_{*}^{i}, x_{i}^{*}\right] \times \mathbb{I} \rightarrow\left[-\delta_{x_{i}}, \delta_{x_{i}}\right]$ of $\xi_{i}(i=1,2, \ldots, q)$. Let $\zeta_{m}: I_{m} \times \mathbb{I} \rightarrow \mathbb{R}$ be a function defined by the formula

$$
\zeta_{m}(z)= \begin{cases}\xi(z) & \text { for } z \in \bigcup_{i=0}^{q}\left[x_{i}^{*}, x_{*}^{i+1}\right] \times \mathbb{I} \\ \xi_{i}^{*}(z) & \text { for } z \in\left[x_{*}^{i}, x_{i}^{*}\right] \times \mathbb{I}(i=1,2, \ldots, q) .\end{cases}
$$

If $I_{m} \cap \bar{D}=\emptyset$, then we put $\zeta_{m}=\xi_{\mid I_{m} \times \mathbb{I}}$. Let $\hat{\xi}=\nabla_{m} \zeta_{m}$ be a combination of compatible functions $\zeta_{m}$ (cf. [3]). Of course, $\hat{\xi}$ is a continuous function and, according to $(5)$ and $(9), \Gamma(\hat{\xi}) \subset V$.

Sufficiency. If $g$ is a continuous function, then (Theorem 1.4.6 of [6]) the proof of sufficiency is trivial. Assume that $D_{g} \neq \emptyset$. Since $\left(\overline{D_{f}} \cup \overline{D_{g}}\right) \times \mathbb{I} \subset \mathcal{Z}(\xi)$, we infer that

$$
D_{f \triangle g} \subset \mathcal{Z}(f \triangle g) .
$$

By $g(x)=\xi(x, 1)$ and (Theorem 1.1.11 of [6]), the image $\xi(L)$ is a connected set for each arc $L \subset \mathbb{R} \times \mathbb{I}$. Therefore $g$ is a Darboux function such that $D_{g} \subset \mathcal{Z}(g)$ and so, according to Lemma $1, g \in \mathcal{A}_{o}(\mathbb{R}, \mathbb{R})$. So, it is sufficient to show that $f \triangle g$ is an almost continuous function and, at the same time, according to Lemma 1, it is sufficient to show that $f \triangle g$ is a Darboux function.

We suppose to the contrary that $f \triangle g$ does not possess the Darboux property. This means that there exists an interval $[a, b] \subset \mathbb{R}(a<b)$ such that $f \triangle g([a, b])=A \cup B$ where $A$ and $B$ are nonempty, disjoint separated sets. Put $A^{\prime}=[a, b] \cap(f \triangle g)^{-1}(A)$ and $B^{\prime}=[a, b] \cap(f \triangle g)^{-1}(B)$. Then $A^{\prime}, B^{\prime}$ are not separated sets. Suppose, for instance, that there exists $a_{0} \in A^{\prime} \cap \overline{B^{\prime}}$. Let $\left\{b_{n}\right\}_{n=1}^{\infty} \subset B^{\prime} \cap(-\infty, b)$ be a sequence such that $b_{n} \searrow a_{0}$. Then $b_{n} \neq a_{0}$ and $a_{0} \in \overline{D_{f}} \cup \overline{D_{g}}$. Consequently, by applying (10), $f \triangle g\left(a_{0}\right)=(0,0) \in A$. According to the separateness of $A$ and $B$, there exists $\varepsilon>0$ such that

$$
K_{\varepsilon} \cap B=\emptyset \text { where } K_{\varepsilon}=(-\varepsilon, \varepsilon) \times(-\varepsilon, \varepsilon) .
$$

It is easy to check $((10))$ that $b_{n} \notin \overline{D_{f}} \cup \overline{D_{g}}(n=1,2, \ldots)$. Fix $n_{o}$. Let $(p, q)$ be a component of $D^{\prime}=\mathbb{R} \backslash\left(\overline{D_{f}} \cup \overline{D_{g}}\right)$ such that $b_{n_{o}} \in(p, q)$. Clearly, $p \in\left[a_{0}, b_{n_{o}}\right] \subset[a, b)$ and $p \in\left(\overline{D_{f}} \cup \overline{D_{g}}\right)=\overline{D_{f \triangle g}}$ and, consequently, by applying $(10), f \triangle g(p)=(0,0)$. By our assumptions, there exists a topology $\tau s$-finer than the topology $\tau_{o}$, such that $f, g:\left(\mathbb{R}, \tau_{o}\right) \rightarrow \mathbb{R}$ are continuous. Let $U$ be a $\tau$-neighborhood of $p$ such that

$$
U \subset(-\infty, b) \text { and } f(U), g(U) \subset(-\varepsilon, \varepsilon) .
$$


We shall show that

$$
U \cap(p, q) \neq \emptyset .
$$

Suppose to the contrary that $U \cap(p, q)=\emptyset$. Then $p \in U \cap(-\infty, q) \subset(-\infty, p]$, which means that $(-\infty, p]=(-\infty, p) \cup(U \cap(-\infty, q))$ is a $\tau$-open set and, consequently, $(p,+\infty)$ is a $\tau$ closed set, which is impossible. From (13) we infer that there exists $y \in U \cap(p, q)$ and, according to (11) and (12),

$$
f \triangle g(y) \in A \text {. }
$$

Since $f \triangle g_{\mid(p, q)}$ is a continuous function, $f \triangle g\left(\left[b_{n_{o}}, y\right]\right)$ is a connected set contained in $f \triangle g([a, b])$ such that $f \triangle g\left(\left[b_{n_{o}}, y\right]\right) \cap A \neq \emptyset$ and $f \triangle g\left(\left[b_{n_{o}}, y\right]\right) \cap B \neq \emptyset$, which contradicts the separateness of $A$ and $B$.

Before going on, we introduce a bit more notation which we shall use. For an arbitrary function $f \in \mathcal{A}_{o}(X, Y)$,

$$
a c H(f)=\left\{g \in \mathcal{A}_{o}(X, Y): f \frac{a c}{\xi} g, \text { for some homotopy } \xi\right\} .
$$

It is not hard to verify that if $f, g, h \in \mathcal{A}_{o}(\mathbb{R}, \mathbb{R})$, then $f \in a c H(f)$ and if $g \in a c H(f)$, then $f \in a c H(g)$. But, in the general case, the ac-homotopies are not transitive relations. In fact, let $f, g$ be functions from the example given at the beginning and let $h$ be an arbitrary continuous function. Since $(([4]),([6])) f \triangle h, g \triangle h \in \mathcal{A}\left(\mathbb{R}, \mathbb{R}^{2}\right)$, Theorem 1 shows that $f \frac{a c}{\xi_{1}} h$ and $h \frac{a c}{\xi_{2}} g$ by the symmetry of ac-homotopies. Of course, $f$ and $g$ are not ac-homotopic.

The following theorems show that the facts considered (diagonals and achomotopies) can be applied to the characterization of continuity or investigations of operations on almost continuous functions.

Theorem 2. Let $f: \mathbb{R} \rightarrow \mathbb{I}$ be an arbitrary function. Then the following conditions are equivalent:

(i) $f$ is a continuous function;

(ii) $f \in \mathcal{A}_{o}(\mathbb{R}, \mathbb{I})$ and $\Delta_{\mathcal{A}}(f)$ is not a porous set at $f$ in the space $\mathcal{A}(\mathbb{R}, \mathbb{I})$;

(iii) $f \in \mathcal{A}_{o}(\mathbb{R}, \mathbb{I})$ and, in the subspace $\mathcal{A}_{o}^{f}(\mathbb{R}, \mathbb{I})=\left\{\eta \in \mathcal{A}_{o}(\mathbb{R}, \mathbb{I}) ; \mathcal{Z}(\eta) \subset\right.$ $\mathcal{Z}(f)\}$ of $\mathcal{A}_{o}(\mathbb{R}, \mathbb{I})$, acH $(f) \cap \mathcal{A}_{o}^{f}(\mathbb{R}, \mathbb{I})$ is not a porous set at $f$.

Proof. First we shall show that $(i) \Rightarrow(i i)$ and $(i) \Rightarrow($ iii $)$. Let $f: \mathbb{R} \rightarrow \mathbb{I}$ be an arbitrary continuous function. According to Theorem 1.4.4 from [6], $f \triangle g \in$ $\mathcal{A}\left(\mathbb{R}, \mathbb{I}^{2}\right)$ for an arbitrary $g \in \mathcal{A}(\mathbb{R}, \mathbb{I})$ and, consequently, $\Delta_{\mathcal{A}}(f)=\mathcal{A}(\mathbb{R}, \mathbb{I})$, which ends the proof of the first implication. Moreover, let $t \in \mathcal{A}_{o}^{f}(\mathbb{R}, \mathbb{I})$. Then $D_{f \triangle t} \subset \mathcal{Z}(t)=\mathcal{Z}(f \triangle t)$ and so (according to the above considerations), 
$t \in \Delta_{\mathcal{A}_{o}}(f)$. Theorem 1 now leads to the relation $f \frac{a c}{\xi} t$ and so, $a c H(f) \cap$ $\mathcal{A}_{o}^{f}(\mathbb{R}, \mathbb{I})=\mathcal{A}_{o}^{f}(\mathbb{R}, \mathbb{I})$.

Now, we shall show that $(i i) \Rightarrow(i)$ and $(i i i) \Rightarrow(i)$. Suppose to the contrary that there exists $x_{0} \in D_{f}$. Then let $\varepsilon>0$ be a number such that $\frac{\varepsilon}{2}$ is a (for instance, right-hand side) cluster number of $f$ at $x_{0}$. Since $x_{0}$ is a right-hand side Darboux point of $f([1],[5])$ for a fixed $\alpha \in\left(\frac{\varepsilon}{4}, \frac{\varepsilon}{2}\right)$, there exists a sequence $\left\{x_{n}\right\}$ such that $x_{n} \searrow x_{0}$ and $f\left(x_{n}\right)=\alpha$. Since $x_{n} \in C_{f}(n=1,2, \ldots)$, for every $n$, there exist $x_{n}^{\prime}, x_{n}^{\prime \prime}$ such that $x_{n+1}^{\prime \prime}<x_{n}^{\prime}<x_{n}<x_{n}^{\prime \prime}$ and

$$
f\left(\left[x_{n}^{\prime}, x_{n}^{\prime \prime}\right]\right) \subset\left(\frac{\varepsilon}{4}, \frac{\varepsilon}{2}\right) \text {. }
$$

Moreover, let $p_{n} \in\left(x_{n}^{\prime}, x_{n}\right), q_{n} \in\left(x_{n}, x_{n}^{\prime \prime}\right)$ for $n=1,2, \ldots$ Now, we define a function $h: \mathbb{R} \rightarrow \mathbb{I}$ in the following way:

$h(x)=0$ for $x=x_{n}(n=1,2, \ldots)$;

$h(x)=f(x)$ for $x \leq x_{0}, x=p_{n}, x=q_{n}(n=1,2, \ldots)$ and $x \geq q_{1}$;

$h(x)=\frac{\varepsilon}{2}$ for $x=x_{n}^{\prime}$ or $x=x_{n}^{\prime \prime}(n=1,2, \ldots)$;

$h(x)$ is linear in the segments $\left[x_{n}^{\prime}, p_{n}\right],\left[p_{n}, x_{n}\right],\left[x_{n}, q_{n}\right](n=1,2, \ldots)$ and $\left[q_{n}, x_{n}^{\prime \prime}\right](n=2,3, \ldots)$;

$h(x)=\max \left(f(x), \frac{\varepsilon}{2}\right)$ for $x \in\left[x_{n+1}^{\prime \prime}, x_{n}^{\prime}\right](n=1,2, \ldots)$.

According to (14), Theorem 1.7.11 and Lemma 1.2.4 from [6], $h$ is an almost continuous function such that $D_{h} \subset\left(D_{f} \cap\left(-\infty, x_{0}\right]\right) \cup\left(D_{f} \cap\left[q_{1},+\infty\right)\right) \subset D_{f}$ and $\mathcal{Z}(h) \supset\left(\mathcal{Z}(f) \cap\left(-\infty, x_{0}\right]\right) \cup\left(\mathcal{Z}(f) \cap\left[q_{1},+\infty\right)\right)$, which means that $h \in$ $\mathcal{A}_{o}^{f}(\mathbb{R}, \mathbb{I})$. Note that $\varrho(h, f) \leq \frac{\varepsilon}{2}$. Now, we consider $B\left(h, \frac{\varepsilon}{8}\right)$ (in the case of our considerations, the symbol $B\left(h, \frac{\varepsilon}{8}\right)$ denotes the open ball in the space $\mathcal{A}(\mathbb{R}, \mathbb{I})$ as well as in the space $\left.\mathcal{A}_{o}^{f}(\mathbb{R}, \mathbb{I})\right)$. It is obvious that $B\left(h, \frac{\varepsilon}{8}\right) \subset B(f, \varepsilon)$. The proof will be completed by showing that

$$
B\left(h, \frac{\varepsilon}{8}\right) \cap \Delta_{\mathcal{A}}(f)=\emptyset
$$

in the space $\mathcal{A}(\mathbb{R}, \mathbb{I})$ and

$$
B\left(h, \frac{\varepsilon}{8}\right) \cap a c H(f)=\emptyset
$$

in the space $\mathcal{A}_{o}^{f}(\mathbb{R}, \mathbb{I})$.

Proof OF (15). Let $g \in B\left(h, \frac{\varepsilon}{8}\right)$. Then

$$
f \triangle g\left(\left[x_{0}, x_{1}\right]\right) \subset\{(0,0)\} \cup\left(\mathbb{R}^{2} \backslash\left(\left[0, \frac{\varepsilon}{5}\right] \times\left[0, \frac{\varepsilon}{5}\right]\right)\right),(0,0) \in f \triangle g\left(\left[x_{0}, x_{1}\right]\right)
$$

and $f \triangle g\left(\left[x_{0}, x_{1}\right]\right) \cap\left(\mathbb{R}^{2} \backslash\left(\left[0, \frac{\varepsilon}{5}\right] \times\left[0, \frac{\varepsilon}{5}\right]\right)\right) \neq \emptyset$, which (according to theorem 1.1.11 of [6]) means that $f \triangle g$ is not an almost continuous function. 
Proof OF (16). Suppose to the contrary that there exists $g \in B\left(h, \frac{\varepsilon}{8}\right) \cap$ $a c H(f)$. Then $g(x)>\frac{\varepsilon}{8}$ for each $x \in\left(x_{0}, x_{1}\right) \backslash \bigcup_{n=1}^{\infty}\left(p_{n}, q_{n}\right)$. Let $\tau$ be a topology $s$-finer than $\tau_{o}$ such that $f, g:(\mathbb{R}, \tau) \rightarrow \mathbb{I}$ are continuous and let $V$ be a $\tau$-neighborhood of $x_{0}$ such that $f(V) \subset\left[0, \frac{\varepsilon}{9}\right) \supset g(V)$. Since $g(V) \subset\left[0, \frac{\varepsilon}{9}\right)$,

$$
V \cap\left(x_{0}, x_{1}\right] \subset \bigcup_{n=1}^{\infty}\left(p_{n}, q_{n}\right) .
$$

On the other hand, $f(V) \subset\left[0, \frac{\varepsilon}{9}\right)$ implies that

$$
V \cap\left(x_{0}, x_{1}\right] \cap \bigcup_{n=1}^{\infty}\left(p_{n}, q_{n}\right)=\emptyset .
$$

This means that $V \cap\left(x_{0}, x_{1}\right]=\emptyset$ and, consequently, $\left(x_{0},+\infty\right)$ is a $\tau$-closed set, which is impossible.

Theorem 3. Let $f, g \in \mathcal{A}_{o}(\mathbb{R}, \mathbb{R})$. If $f \triangle g \in \mathcal{A}_{o}\left(\mathbb{R}, \mathbb{R}^{2}\right)$, then $f+g, f \cdot g$, $\min (f, g)$, and $\max (f, g) \in \mathcal{A}_{o}(\mathbb{R}, \mathbb{R})$.

Proof. We give the proof only in the case $f+g$. Assume that $f \triangle g \in$ $\mathcal{A}_{o}\left(\mathbb{R}, \mathbb{R}^{2}\right)$. Let $h: \mathbb{R}^{2} \rightarrow \mathbb{R}$ be defined by $h(x, y)=x+y$. Then $h$ is continuous, so $f+g=h \circ(f \triangle g)$ is an almost continuous function (see [8] or [6]). Since $D_{f+g} \subset D_{f} \cup D_{g}=D_{f \triangle g},(f+g)(x)=0$ for each $x \in D_{f+g}$. Thus $f+g \in \mathcal{A}_{o}(\mathbb{R}, \mathbb{R})$.

\section{References}

[1] A. Bruckner and J. Ceder, Darboux continuity, Jbr. Deutsch. Math. Verein 67 (1965), 93-117.

[2] J. B. Brown, Almost continuous Darboux functions and Reed's pointwise convergence criteria, Fund. Math. 86 (1974), 1-7.

[3] R. Engelking, General topology, Polish Scientific Publishers (PWNWarszawa) (1977).

[4] J. Jastrzȩbski, J. Jędrzejewski and T. Natkaniec, On some subclasses of Darboux functions, Fund. Math. 138 (1991), 165-173.

[5] J. S. Lipiński, On Darboux points, Bull. Acad. Pol. Sci. 26.11 (1978), 869-873. 
[6] T. Natkaniec, Almost continuity, habilitation thesis, Bydgoszcz (1992), $1-131$.

[7] R. J. Pawlak, Darboux homotopies and Darboux retracts - results and questions, Real Analysis Exch. 20 (1994-95), 805-814.

[8] J. Stallings, Fixed point theorem for connectivity maps, Fund. Math. 47 (1959), 249-263.

[9] L. Zajíček, Sets of $\sigma$-porosity and sets of $\sigma$-porosity (q), Časopis Pěst. Mat. 101 (1976), 350-359.

[10] L. Zajíček, Porosity and $\sigma$-porosity, Real Analysis Exch. 13 (1987-88), $314-350$. 